\title{
THEORY OF CIRCUMSTELLAR ENVELOPES
}

\author{
R. Wehrse \\ Institut $f$. Theoretische Astrophysik \\ Im Neuenheimer Feld 561 \\ D 6900 Heidelberg \\ Federal Republic of Germany
}

\begin{abstract}
After a comparison of conventional photospheres with circumstellar envelopes the radiation in spherical shells is considered. We discuss the transfer equation and a new quasi-exact solution in term of the transition matrix. Various methods used for the numerical evaluation of the specific intensities are summarized. The general properties of the radiation fields and some recent detailed model calculations are briefly reviewed.
\end{abstract}

\section{INTRODUCTION}

In the usual nomenclature "circumstellar envelope" or "circumstellar shell" designates the space around a star, which is outside the star's atmosphere, but where the matter is still connected to the star. Although there is evidently no strict separation between outer layers of the atmosphere and the circumstellar envelopes, both have quite different characteristics, the most important being the different geometry and the different energy as well as momentum balance. In all cases this means a much more complicated behaviour of the circumstellar envelopes. Therefore, it has not yet been possible to construct realistic models for circumstellar envelopes from first principles and without severe simplifications.

In Table 1 we 1 ist some details, but it is beyond the scope of this paper to present a complete review of the field, since recently excellent and detailed papers have appeared summarizing the present knowlegde e.g. on the physical and chemical state of matter in the envelopes around cool stars (Omont, 1985 ; Gail and Sedlmayr, 1986 ), the mass loss mechanisms for hot and cool stars ( Hearn, 1987, Holzer, 1987 ), etc..

We will focus on the radiative transfer in circumstellar 
$\underline{T}$ a b 1 e 1

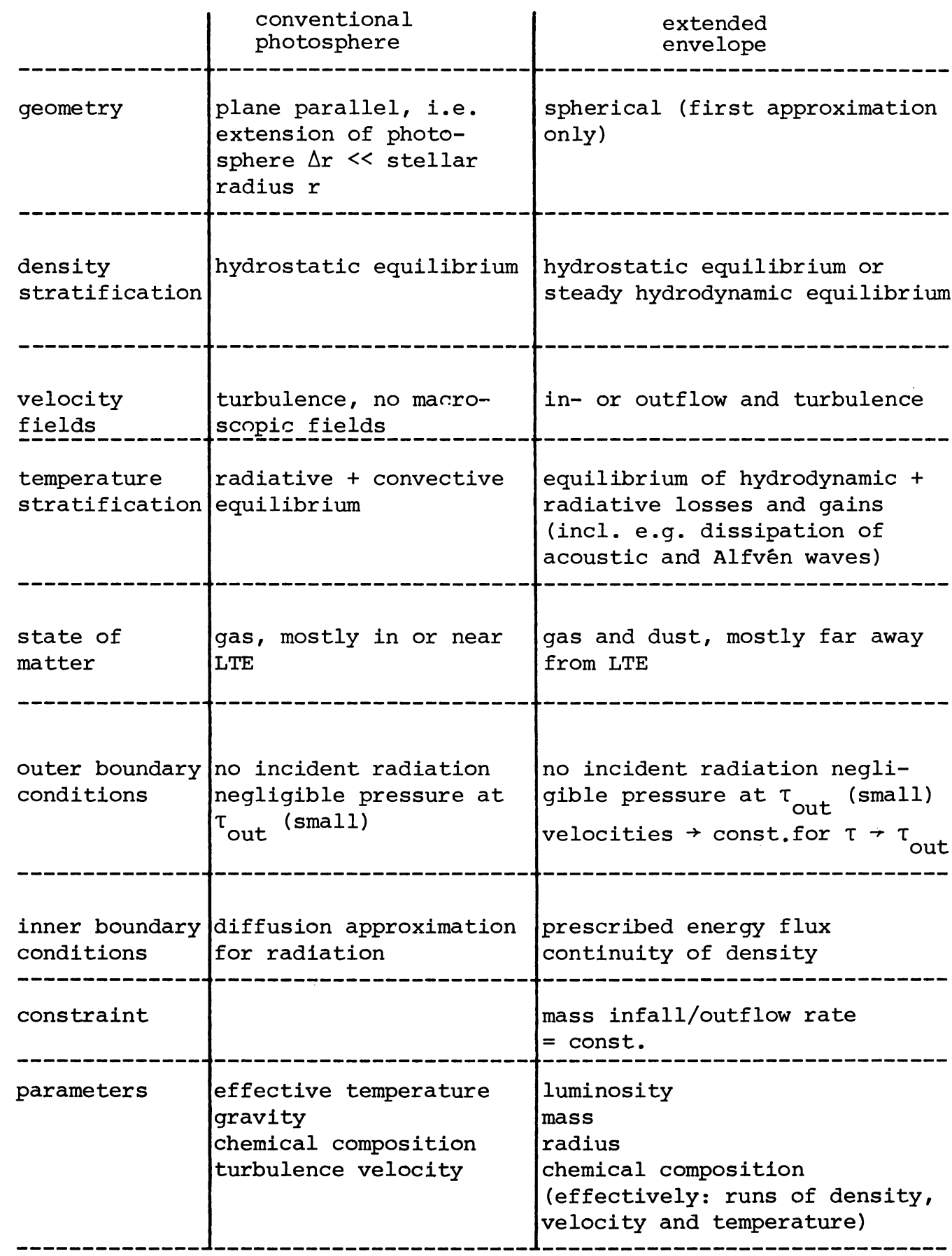


shells, which requires a more sophisticated treatment than in stellar atmospheres, because (i) in addition to turbulence velocity fields with large scales ( in most cases radial flows, have to be taken into account; (ii) the geometrical extension of the configuration has to be considered explicitly; ( $i$ ii) radiative processes dominate over collisional processes so that the absorption and scattering coefficients are coupled directly to the radiation field (and not only via the energy equation).

In Section 2 the radiative transfer equation for spherical configurations with radial velocitiy fields is given and various methods for its solution are described. The resulting characteristics of the radiation fields are summarized in Section 3. Finally, in Section 4 we discuss briefly some recent calculations modeling the envelopes of cool giants, Be stars, and supernovae.

\section{THE RADIATIVE TRANSFER EQUATION FOR SPHERICALLY EXTENDED} CONFIGURATIONS AND ITS SOLUTION

We will consider subsequently spherical configurations, because for them methods for the evaluation of the radiation field are well established, although no standards have emerged yet ( cf. Beckman and Crivellari, 1985). This restriction to spherical geometry implies that we cannot discuss polarisation effects and that we have to exclude binary stars, discs, and jets. On the other hand, speckle interferometry ( Roddier, Roddier, and Karovska, 1985) indicates that the distribution of matter around most single stars is approximately spherical and we therefore expect that the majority of lines originating in the shells of these objects are well described by this approximation. For exceptions at highest luminosities see Wolf's contribution at this conference. In addition we will neglect time dependent and stochastic effects (for the latter see e.g. Traving, 1975, Gierens, Traving, and Wehrse, 1986, or Albrecht, 1986 ).

The equation for the stationary transport of unpolarized radiation can be written

(i) in the Eulerian or observer's frame (see e.g. Mihalas, 1978, or Cannon, 1985):

$$
\left(\mu \frac{\partial}{\partial r}+\frac{1}{r}\left(1-\mu^{2}\right) \frac{\partial}{\partial \mu}\right) I=-(x+\sigma) I+(x+\sigma) S
$$

with

$$
\begin{aligned}
\mu & =\text { cosine between the normal and the ray direction } \\
r & =\text { radial coordinate } \\
I & =\text { specific intensity }
\end{aligned}
$$




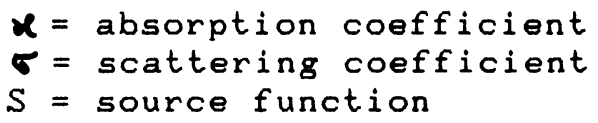

or

(ii) in the Lagrangian or comoving frame

$\left\{\mu_{0} \frac{\partial}{\partial r}+\frac{1}{r}\left(1-\mu_{0}^{2}\right) \frac{\partial}{\partial \mu_{0}}-\frac{\nu_{0} v}{c r}\left[\left(1-\mu_{0}^{2}\right)+\mu_{0}^{2} \frac{\partial \ln v}{\partial \ln r} \frac{\partial}{\partial v_{0}}\right] I_{0}=\left(x_{0}+\sigma_{0}\right)\left(S_{0}-I_{0}\right)\right.$

with

$$
\begin{aligned}
& \boldsymbol{v}=\text { radial velocity } \\
& \boldsymbol{v}=\text { frequency. }
\end{aligned}
$$

The subscript 0 indicates quantities in the comoving frame. For comparison with observations they have to be transformed to the rest frame.

The absorption and the scattering coefficients as well as the source function depend on radius, the angle coordinate, the frequency, the temperature, mean intensities, and particle occupation numbers. Whereas in comoving frame calculations the phase function is the only source of the direction dependence and can in most cases be neglected, in observer's frame computations all these quantities are strongly anisotropic because in addition the Doppler effect

$$
v=v_{0}(1 \pm \mu v / c)
$$

has explicitly to be taken into account. On the other hand, this is at least partly compensated by the fact that the observer's frame equation is considerably simpler.

The choice of the coordinate frame is determined by numerical (and perhaps personal) conveniance; the physics to be considered ( e.g. complete or partial redistribution) must in both cases be identical.

There is no general analytical solution of the partial integro differential equations (1) or (2) known. However, they can easily be solved if they are transformed to a system of ordinary differential equations by discretizing the angle-frequency space, i.e. by considering the specific intensity $I$ and the source function $S$ as vectors

$$
\begin{aligned}
& I=\left(I_{1}, I_{2}, I_{3}, \ldots \ldots, I_{n}\right)^{t} \\
& S=\left(S_{1}, S_{2}, S_{3}, \ldots, S_{n}\right)^{t}
\end{aligned}
$$

where $n$ is the numbers of angles times the numbers of frequencies. There are presently two schemes used for the discretisation of the angle space (Fig. 1): (i) rays in the configuration space, i.e. the integration follows the propagation of the light. This discretisation has the advantage that the peaking effect (see below) is 


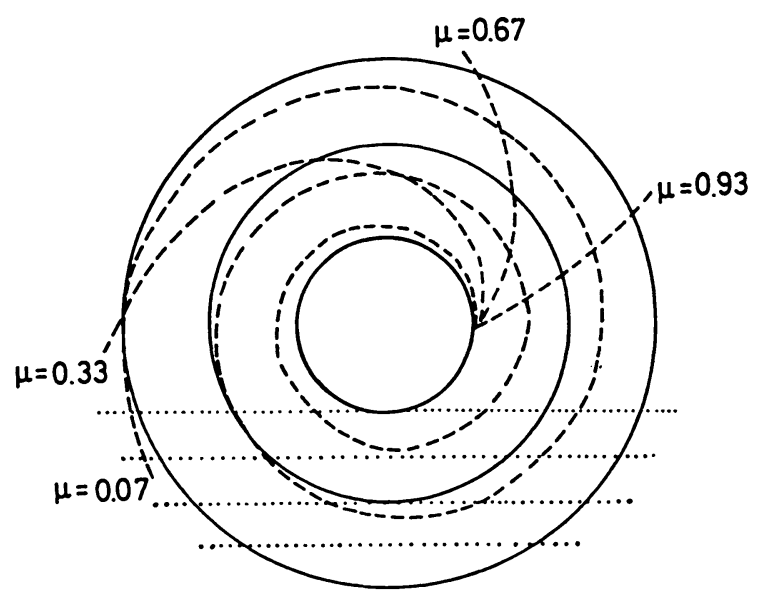

Fig. 1. Discretisation schemes for spherical radiative transfer equations. Dotted 1 ines indicate light rays with "impact" parameters $p=1.0,1.5,2.0$, and 2.5. The logarithmic spirals represent the discretisation $\mu(r)=$ const for a Gaussian 4 point division.

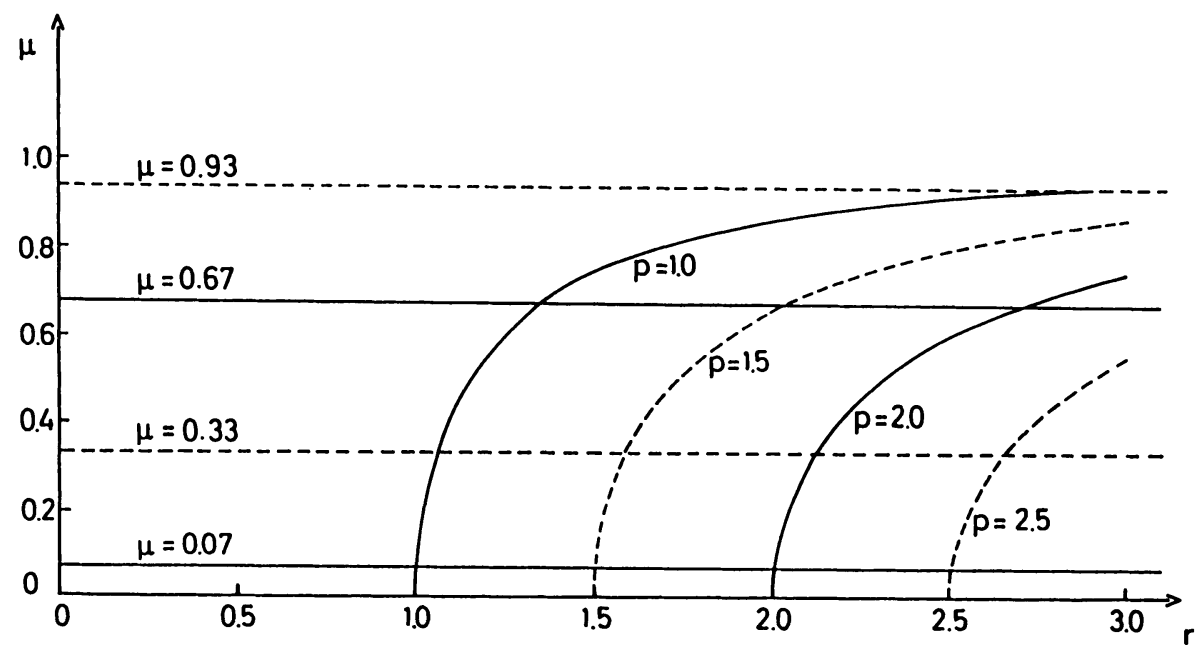

Fig. 2. Representation of the same discretisation schemes as in Fig. 1, but in the $r \times \mu$ space. The horizontal lines give $\mu(r)=$ const for the Gaussian division and the curves indicate the light rays. 
well represented. It has the disadvantage that the angles to the radial direction vary with $r$ and therefore makes divergence-free formulations impossible.

(ii) constant angles to the normal direction. The corresponding curves in the configuration space are logarithmic spirals. With this discretisation a divergence-free form of the transfer equation can be constructed ( Grant and Peraiah, 1972, Peralah, 1984, and for a Gaussian division in $\mu$ the numerical accuracy is already very high for a rather small number of number of angles, if the radiation does not peak too much. Such a discretisation is therefore particularly advantageous for problems of high optical depths and small to moderate geometrical extensions.

Note that both discretisations must be considered equally "natural", since it depends only on the mapping whether they are represented by straight lines ( the first one in the configurations space, the second one in the $r \times \mu$ space, see Fig. 2 ) or by curves ( $r \times \mu$ space for the first one, configuration space for the second one).

Both forms of the transfer equation (eqs. 1 and 2 ) can now be written in matrix notation

$$
\frac{d}{d r} I_{r}=A_{r} I_{r}+Q_{r}
$$

where the coupling matrix $A$ contains all terms proportional to $I$ and $Q$ describes the photon sources.

Now the solution can be expressed in terms of the transition matrix

$$
I_{r}=\phi_{r, 0} I_{0}+\int_{0}^{r} \phi_{r, r^{\prime}} Q_{r^{\prime}} d r^{\prime}
$$

The transition matrix $\phi$ is defined by the matrix differential equation ( see Bronson,1970)

$$
\frac{d}{d r^{\prime}} \phi_{r, r^{\prime}}=A \phi_{r, r^{\prime}}
$$

with the initial condition

$$
\phi_{r_{1}^{\prime}, p^{\prime}}=1
$$

This formalism for the solution of a system of differential equations is frequently used in quantum electrodynamics ( cf.e.g. Louisell, 1973 , , but to my knowledge has not yet been employed in radiative transfer theory. The main reason seems to be that eq. 6 hardly can be used in numerical 
calculations because in the form given above it contains terms that increase exponentially with the optical depth and can hardly be manipulated by a computer ( however, see below). Therefore, either the transfer equation or the solution has first to be transformed analytically into a form suitable for numerical evaluations. In the literature a large variety of such transformations are found; they can be summarized in the following way ( see also Kunasz, 1985 ):

a) one-step-solutions They are possible if the source function is known or it can be expressed as a linear function of the specific intensity. This is e.g the case for line radiation from a two-level-atom, where

$$
S=\varphi^{-1}\left\{(1-\varepsilon) \iint R I d \mu^{\prime} d v^{\prime}+\varepsilon B\right\}
$$

(Mihalas, 1978 ). Then the differential equation for the transition matrix can be transformed into differential equations for the transmission and reflection matrices ( Schmidt and Wehrse, 1987 ). The system may be stiff, but the corresponding solutions contain only decreasing and weakly inreasing terms. Although this method in many cases may not be economical due to the large matrices involved and the small integration step-size required it provides a way for obtaining quasi-exact solutions of the general problem. If $A$ and/or $Q$ have a simple structure or - on physical grounds can be simplified - the solution of eq. can be obtained by quadratures, often even analytical expressions can be given. This is e.g. the case if the depth dependence of the extinction coefficients and of the source function can be approximated by polynomials (Schmid-Burgk, 1975) or if the $l$ ine profile is narrow and the radial velocity field has a large gradient (Sobolev, 1960).

b) iterative methods

These methods, which may be used in parallel with the solution of the rate equations, may be divided into the following groups:

(i) moment methods, in which differential equations for the angle-moments of the specific intensity are solved with closure relations that are updated

iteratively. The most well-known method of this group is the Feautrier-methods with variable Eddington factors ( Mihalas, 1978 ).

(ii) Newton-Raphson iterations, in which the transfer equation and the equation determining the source function are paramterized ( or discretized) with respect to depth and solved by linearisation ( i.e. a Newton Raphson method ).

(iii) operator perturbation methods, in which the matrix $A$ ( which is considered as an operator) is written as 


\section{$A=A_{0}+A_{1}$}

and $A_{1}$ is considered to be a perturbation. The solution then proceeds in complete analogy to the Dyson expansion in quantum electrodynamics ( Dyson, $1949 \mathrm{a}, \mathrm{b}$ ). Note that for the continuous depth space the iteration converges, if only all coefficients are bounded, but that in the discrete space in general the differentiation operator is also perturbed and then for convergence also the condition

$$
\left\|1-A_{1}^{-1} A_{0}\right\|<1
$$

is required ( Kalkofen, 1985 ). This method has recently become very popular because decompositions of $A$ have been devised (Scharmer, 1984 ) in which the zero order term contains already the largest part of the information and still can be very quickly evaluated.

(c) Monte Carlo methods

In this method the paths of individual photons are followed and the specific intensity is determined a posteriori by photon statistics; for an example see Lee and Meier, 1980. Complicated redistribution functions and/or deviations from sphericity can easily be included, but as for the one-step-methods the source function must begiven explicitely or by an expression linear in the specific intensity. For reaching a high accuracy this method is in many cases very time consuming.

In addition to the "pure" methods summarized above, various combinations e.g. in perturbation approaches are possible and have been attempted.

\section{SOME CHARACTERISTICS OF SPHERICAL RADIATIVE FIELDS}

The radiation fields in spherical configurations differ from those in plane-parallel geometry in many respects:

(i) The primary difference is the dilution of the field, i.e. the fact that for a conservative system the flux is proportional to $r^{2}$, whereas for a plane-parallel medium it is constant. In numerical calculations this effect can easily be taken into account by replacing the intensity $I$ by $I^{\prime}=4 \pi r^{2} I$, since the transfer equation keeps its form under this transformation. Although it is often rather small ( e.g. in red giant atmospheres withAr/r $\approx 1 . e-2$ ) it may have severe consequences, for instance by "switching on" the formation of molecules like $\mathrm{H}_{2} \mathrm{O}$, which absorb strongly and change the temperature structure in the optically thin layers. 
(ii) If a photon in a sphere travels outwards ( without interactions, its angle to the normal decreases contiuously ("peaking effect", Fig. 1 ). It is particularly important, if the medium is optically thin and geometrically very extended. In the transfer equation the second term on the left hand side takes care of this effect. Since an accurate discrete representation of this term is inhibited by the requirement that no photons should be generated or lost by the numerical evaluation of the source term, it mainly causes the complications of spherical radiative transfer ( for details see Schmidt and Wehrse, 1987).

(iii) The escape probability of photons travelling in directions other than the normal one is larger since for them the optical depth to the surface is lower than in plane parallel geometry. This implies that the mean intensity cannot build up as high in spherical geomtries and therefore the radiation pressure ( in particular from lines) and fluorescence efficiences are smaller.

(iv) In a sphere all radial velocity fields lead to velocity gradients ( at least in the transversal direction) and affect directly and in a depth dependent way the radiation field whereas in slabs $v(r)=$ const. only means a global Doppler shift.

(v) Since the continuous absorption is usually weak in circumstellar shells, for small velocities often the lines are extremely optically thick and therefore deviations from complete redistribution become significant (Hubeny, 1985).

\section{SOME EXAMPLES FOR MODEL CALCULATIONS}

The most significant effects of spherical radiative transfer are found for cool giants and supergiants since many of these stars have extended photospheres ( Watanabe and Kodaira, 1978, 1979; Schmid-Burgk, Scholz and Wehrse, 1981) and are surounded by huge envelopes. In the photospheres of luminous $M$ stars the geometrical extension "switches on" the formation of water vapor which makes the outer parts several hundert degrees cooler than expected from plane parallel models. This temperature decrease shows up in the depths of the molecular lines and bands.

Main diagnostic tools for these outer layers are CO lines, since a) they show up in all cool giants; b) they form both in the outer photosphere $(\Delta v=2$ lines mainly with $\lambda \approx$ 2. $3 \mu$, see e.g Höflich et al., 1986) and in the envelope ( $\Delta u=1$ lines with $\lambda \approx 4.5 \mu$, cf. Sahai and Wannier, 1985 ) and in both spectral ranges lines of rather different excitation potential are visible; c) accurate transition probabilities are available (Tipping, 1976); and d) the profiles can be well observed by means of Fourier transform spectroscopy (Maillard, 1974, Hinkle, 1978). 
By fitting such $\mathrm{CO} \Delta \boldsymbol{v}=2$ profiles from Her Höflich et al. ( 1986 ) can show that the 1 ines are formed in LTE and that the photosphere and the envelope must be separated by a chromosphere in which the CO molecule is destroyed. The $\Delta \boldsymbol{v}=$ 1 emission lines from the envelope of the carbon rich object IRC +10216 have been studied in detail by Sahai and Wannier ( 1985 ). Using radiative transfer calculations in the Sobolev approximation (1960) and statistical equilibrium level populations for several rotation lines of the $P$ and the $R$ branch and taking into account different apertures they are able to derive from the observed line strengths reliable information on the temperature distribution and the mass loss rate.

Whereas for these cool stars the photosphere and the envelope can be calculated separately due to the small Rosseland opacity of the shell, this is no longer possible for hotter stars in which the envelope is ionized: By physically consistent NLTE models for Be stars Höflich ( 1986 ) demonstrates that level occupations and the temperature structure in the photosphere are strongly influenced by the density in the shel1. If he takes this effect fully into account, he is able to reproduce the line strenghts and profiles, Balmer jumps etc. of all Be stars, for which reliable data have been published, with a smaller number of free parameters than previously considered to be necessary.

An example for an unexpected result is provided by the atmosphere of a supernova type II atmosphere during the coasting phase, which may be considered as an extreme circumstellar envelope: Although the density is very low and Thomson scattering by far prevails over absorption, the continuum is formed in LTE ( Höflich, Wehrse, and Shaviv, 1986 ). The cause for this unusual behaviour is that under these conditions the electron scattering is so strong that the radiation field becomes essentially local again, as could be shown by test calculations in which the Thomson cross-section was artificially decreased and the level departure coefficients immediately increased.

These few examples show that spectral features calculated from present day models for spherical envelopes can successfully be used to interpret observations. On the other hand, our knowledge on the physics of such regions ( in particular the hydrodynamics and its interaction with the thermodynamics and the radiation field, is still rather limited since simple concepts are missing and even with a large computer it is today just possible to calculate for a given velocity distribution the radiation field and the occupation numbers of a few levels ( $\leqslant 100$ ) consistently. Fortunately, this also means that much better models and new effects can be expected in near future, when a new generation of machines becomes available. 
Acknowledgement: This work was supported by the Deutsche Forschungsgemeinschaft ( SFB 132 ).

\section{References}

Albrecht, M. : 1986 , Ph.D. Thesis, Frankfurt University. Beckman, J.E., Crivellari, L., eds.: 1985, Progress in Stellar Spectral Line Formation Theory, Reidel, Dordrecht.

Bronson, R. : 1970 , Matrix Methods, Academic Press, New York.

Cannon, C.J.: 1985, The Transfer of Spectral Line Radiation, Cambridge University Press, Cambridge.

Dyson, F.J.: 1949a, Phys. Rev. 75, 486.

Dyson. F.J. : $1949 \mathrm{~b}, \overline{\text { Phys. }}$ Rev. $75,1736$.

Gail, H.P., Sedlmayr, E.: 1986 , Proc. Irsee Conf. on Interstellar Matter, in press.

Gierens, K.M., Traving, G., Wehrse, R.: 1986, J. Quant. Spectrosc. Radiat. Transfer, in press.

Grant, I.P., Peraiah, A. : 1972, Mon. Not. R. astr. Soc., 160,321 .

Hearn, A.G.: 1987 , this volume.

Hinkle, K. : 1978, Astrophys. J. 220, 210.

Höflich, P.A.: 1986 , Ph.D. Thesis, Heidelberg University. Höflich, P.A., Wehrse, R., Shaviv, G.: 1986, Astron. Astrophys. 163, 105.

Höflich, P.A., Lowe, R.P., Moorhead, J., Scholz, M. , Wehlau, W. , Wehrse, R. : Mon. Not. R. astr. Soc. 220, 377.

Holzer, T. : 1987 , this volume.

Hubeny, I. : 1985, in: Progress in Stellar Spectral Line Formation Theory, Beckman, J.E., Crivellari, L., eds., Reidel, Dordrecht, p. 27.

Kalkofen, W. : 1985, in: Progress in Stellar Spectral Line Formation Theory, Beckman, J.E., Crivellari, L., eds., Reidel, Dordrecht, p. 153.

Kunasz, P.B. : 1985, in: Progress in Stellar Spectral Line Formation Theory, Beckman, J.E., Crivellari, L., eds., Reidel, Dordrecht, p. 319.

Lee, J.-S., Meier, R.R. : 1980, Astrophys. J. 240, 185.

Louisell, W.H. : 1973 , Quantum $\frac{\text { Statistical Properties of }}{\text { Ptew }}$ Radiation, J. Wiley \& Sons, New York.

Maillard, J.P. : 1974, Highlights of Astronomy 3, 269.

Mihalas, D. : 1978, Stellar Atmospheres, W.H. Freeman, San Francisco.

Omont, A. : 1985, in: Mass Loss from Red Giants, Morris, M. , Zuckerman, B., eds., Reidel, Dordrecht, p. 269.

Peraiah, A.: 1984, in: Methods in Radiative Transfer, Kalkofen, W., ed., Cambridge Universty Press, Cambridge, p. 281 .

Roddier, F., Roddier, C., Karovska, M. : 1985, in: Mass Loss from Red Giants, Morris, M., Zuckerman, B., eds., 
Reidel, Dordrecht, p. 63.

Sahai, R., Wannier, P.G. : 1985, Astrophys. J. $299,424$. Scharmer, G.B.: 1984 , in: Methods in Radiative Transfer,

Kalkofen, W., ed., Cambridge University Press,

Cambridge, p. 173.

Schmid-Burgk, J. : 1975, Astron. Astrophys. 40.249.

Schmid-Burgk, J., Scholz, M., Wehrse. R.: 1981 , Mon.

Not. astr. Soc. 194,383 .

Schmidt, M., Wehrse, R. : 1987, in: Numerical Methods in

Radiative Transfer, Kalkofen, W., ed., Cambridge

University Press, Cambridge, in press.

Sobolev, V.: 1960, Moving Envelopes of Stars, Harvard

University Press, Cambridge/Mass. (Russian Edition 1947).

Tipping, R.H. : 1976, J. molec. Spectrosc. 61, 272.

Watanabe, T., Kodaira, K.: 1978, Publ. Astr. Soc. Japan, 30,21 .

Watanabe, T., Kodaira, K. : 1979, Publ. Astr. Soc. Japan, 31,61 . 